\title{
Multimodal Training Reduces Fall Frequency as Physical Activity Increases in Individuals With Parkinson's Disease
}

\begin{abstract}
Amanda L. Penko, Jacob E. Barkley, Anson B. Rosenfeldt, and Jay L. Alberts
Background: Parkinson's disease (PD) results in a global decrease in information processing, ultimately resulting in dysfunction executing motor-cognitive tasks. Motor-cognitive impairments contribute to postural instability, often leading to falls and decreased physical activity. The aim of this study was to determine the effects of a multimodal training (MMT) versus singlemodal (SMT) training on motor symptoms, fall frequency, and physical activity in patients with PD classified as fallers. Methods: Individuals with PD were randomized into SMT $(n=11)$ or MMT $(n=10)$ and completed training 3 times per week for 8 weeks. The SMT completed gait and cognitive training separately, whereas MMT completed gait and cognitive training simultaneously during each 45-minute session. Physical activity, 30-day fall frequency, and PD motor symptoms were assessed at baseline, posttreatment, and during a 4-week follow-up. Results: Both groups exhibited significant $(P<.05)$ improvements in clinical ratings of motor function, as symptoms improved by $8 \%$ and 15\% for SMT and MMT, respectively. Physical activity significantly increased $(P<.05)$ for both groups from baseline (mean steps 4942 [4415]) to posttreatment (mean steps 5914 [5425]). The MMT resulted in a significant 60\% reduction in falls. Conclusions: Although SMT and MMT approaches are both effective in improving physical activity and motor symptoms of PD, only MMT reduced fall frequency after the intervention.
\end{abstract}

Keywords: dual task, exercise, neurology, gait

Parkinson's disease (PD) is the second most common movement disorder estimated to affect nearly 7 million individuals worldwide. ${ }^{1}$ The loss of dopaminergic neurons within the basal ganglia is the hallmark of PD and manifest in a clinical presentation of motor deficits (ie, postural instability and gait dysfunction) and cognitive dysfunction, as there is a global decrease in information flow and processing. ${ }^{2,3}$ The degeneration of the basal ganglia ultimately result in the clinical presentation of the cardinal motor symptoms of bradykinesia, tremor, rigidity and postural instability and gait dysfunction, and nonmotor symptoms such as cognitive dysfunction. ${ }^{4}$ PD motor and nonmotor symptoms are often exacerbated under dual-task conditions, or the simultaneously completion of 2 tasks, as motor task automaticity is compromised and the addition of a secondary task results in decrements in motor and/or cognitive performance. ${ }^{2,3,5}$

Postural instability and gait dysfunction associated with PD are described as shuffling, small steps, decreased arm swing, and stooped posture, which disturb mobility and postural stability, leading to an increased fall risk. ${ }^{4,6}$ Greater than $50 \%$ of individuals with PD report falling within the previous 2 years, of which $65 \%$ result in injury requiring medical attention. ${ }^{7}$ Falls are often cited as a leading cause for loss of independence in patients with PD. ${ }^{8,9}$ Despite the prevalence of postural instability and gait dysfunction, current pharmacological and surgical interventions are relatively insensitive in alleviating these symptoms. ${ }^{10}$ Thus, a fundamental gap exists in the effective treatment and prevention of falls in patients with PD.

In addition to injury, falling reduces engagement in physical activity behavior in individuals with PD. ${ }^{11}$ An overall reduction in physical activity may have significant implications for patients with

Penko, Rosenfeldt, and Alberts are with Cleveland Clinic, Cleveland, OH. Barkley is with Kent State University, Kent, OH. Penko (penkoa@ccf.org) is corresponding author.
$\mathrm{PD}$, as diminished levels of physical activity are associated with increases in all-cause morbidity and mortality risk. ${ }^{12}$ Individuals with mild-moderate PD accumulate approximately 50\% fewer steps per day than their healthy peers, as they average only 4700 steps per day. ${ }^{13,14}$ The National Health and Nutrition Examination Survey found that by simply replacing 30 minutes per day of sedentary activity with light physical activity, older adults exhibited a $20 \%$ reduction of overall mortality risk, ${ }^{12}$ even if physical activity guidelines were not met. Furthermore, in PD, there is evidence indicating that participation in physical activity provides therapeutic benefits in terms of reducing motor symptoms. ${ }^{15-17}$

In PD, impairments in motor function only partially explain diminished postural function and physical activity behavior as cognitive impairment likely contributes to sedentary behavior and postural instability. This is evident as individuals with PD have greater gait dysfunction under dual-task conditions (eg, carrying on a conversation while walking), where both motor and cognitive processes are active. ${ }^{18-20}$ Gait and posture should be an automated motor task requiring little attentional resources, but the loss of automaticity in PD requires additional cognitive resources dedicated to mobility. ${ }^{5}$

The greater dual-task gait interference in individuals with PD compared with healthy adults is likely related to the disruption of basal ganglia function in the integration of information and the monitoring of voluntary movement and cognitive processes..$^{4,21}$ The basal ganglia contains parallel motor and cognitive neural pathways, and in PD, the selective loss of dopaminergic projections results in motor and cognitive tracts overlapping in the striatum placing individuals at a deficit for neural resources available to successfully complete concurrent motor-cognitive task. . $^{3,22}$

To improve performance under dual-task constructs, motorcognitive training is warranted. Single-modal training involves training motor and cognitive components separately; multimodal training involves training motor and cognitive components simultaneously. The aim of this pilot study was to determine the effects 
of a single-modal (ie, single-task) or multimodal (ie, dual task) physical intervention on motor symptoms, fall frequency, and physical activity, as measured by steps taken, in patients with PD classified as fallers. It was hypothesized that both the singlemodal and multimodal training groups would improve motor symptoms, decrease fall frequency, and increase physical activity behavior; however, only multimodal training group would maintain improvements long term. Rationale for the latter portion of the hypothesis is due to the assumption that multimodal training is more complex, which may provide the environment for motor skill acquisition and retention greater than single-modal training. ${ }^{23,24}$

\section{Methods}

\section{Participants}

A total of 21 participants between the ages of 43 and 77 years (mean age 61.8 [8.8] y) with mild-moderate idiopathic PD were recruited from the Cleveland Clinic (Cleveland, $\mathrm{OH}$ ) and the surrounding area to participate in this pilot randomized trial. The study flow is provided in Figure 1. Sample size validation was computed through an a priori power analysis using Tudor-Locke et al, ${ }^{13}$ meta-analysis examining changes in steps per day in special populations after exercise interventions. Power was set at 0.80 , with $\alpha=.05$. Given the effect size from Tudor-Locke and an alpha of .05, a sample size of 20 would be needed to achieve a power $\geq 0.80$. Therefore, a sample of 21 was deemed to be adequate.

Inclusion criteria consisted of a clinical diagnosis of PD, Hoehn and Yahr stages 2-4, a history of at least 2 falls within the last 12 months, and ability to ambulate a minimum of $300 \mathrm{ft}$ with or without the use of assistive walking device. Exclusion criteria consisted of any musculoskeletal contraindication to exercise, a history of neurological disease other than PD, $\geq 3$ errors on the Short Portable Mental Status Questionnaire, ${ }^{25}$ inability to follow 2-step commands, uncontrolled cardiovascular risk factors classifying the individual as a high-risk exerciser per the American College of Sports Medicine, ${ }^{26}$ and having undergone any surgical procedure for the treatment of PD (eg, deep brain stimulation). Participants were initially phone screened to ensure whether the inclusion and exclusion criteria were met, and medical records were obtained to verify PD diagnosis. All participants read and signed an informed consent document that was approved by the

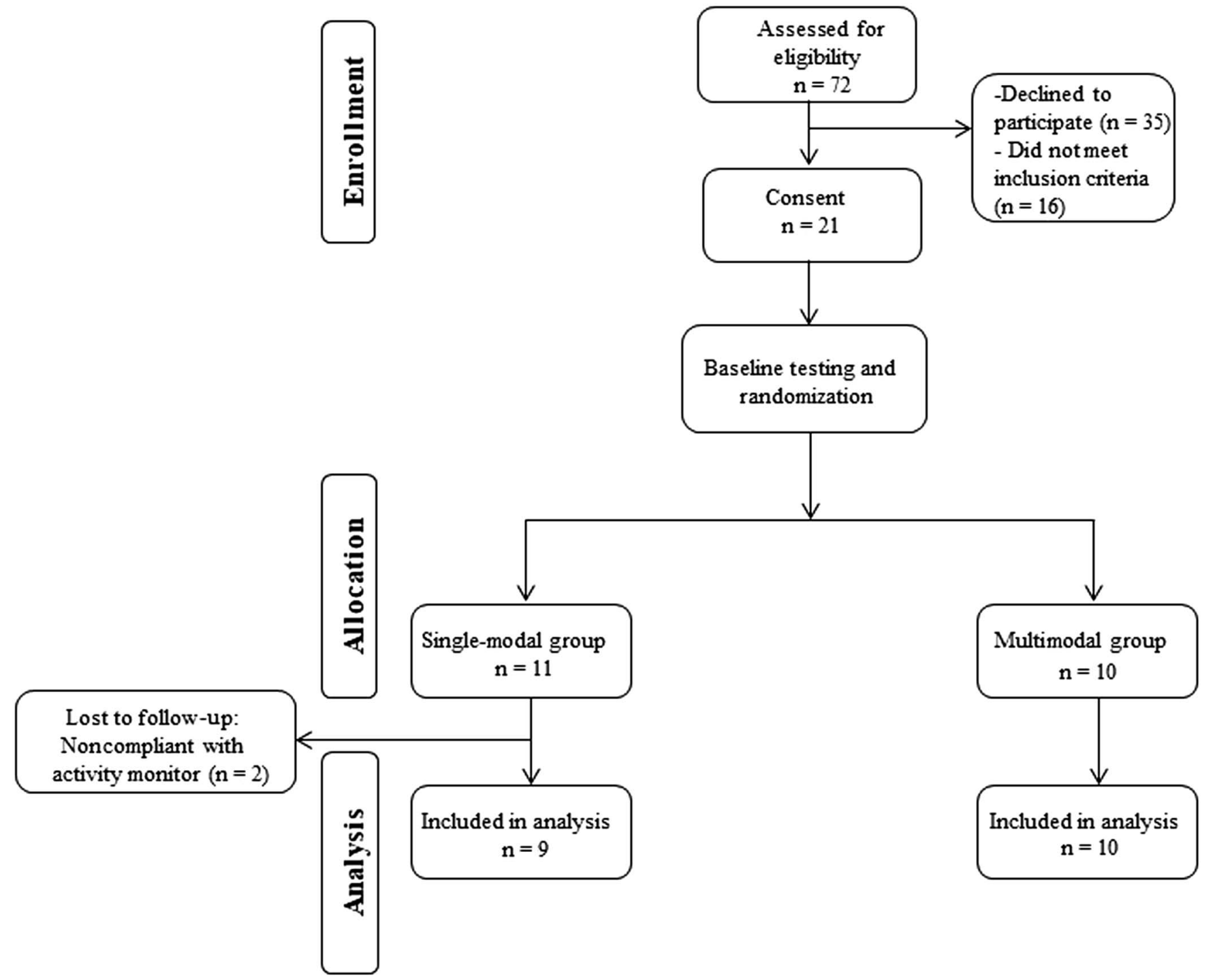

Figure 1 - Study flow diagram. 
Cleveland Clinic Institutional Review Board prior to the start of any study activity.

\section{Intervention}

Participants were randomized into either a single-modal or a multimodal intervention group. All training was supervised by a licensed physical therapist who specialized in neurological rehabilitation at the main campus of the Cleveland Clinic. Both singlemodal and multimodal training were administered 3 times per week for 45-minute sessions and for 8 weeks. The single-modal group evenly divided the 45-minute session between gait training and cognitive training during each session. Each task (ie, gait and cognitive) was preformed separately focusing on 1 task at a time. The dual-task group consisted of 45 minutes of gait training while simultaneously completing a cognitive task. Cognitive training tasks were identical for both groups and involved executive function, attention, memory, and language tasks. Gait training in both groups focused on improving the quality of gait (eg, gait velocity, step length).

\section{Clinical and Physical Activity Assessment}

Parkinson's disease motor symptoms, fall frequency, and physical activity assessments were completed at 3 time points: baseline (ie, 1 wk preceding the intervention); postintervention (ie, within 1 wk following intervention); and 4-week postintervention. For PD motor symptoms assessment, participants were instructed to take their antiparkinsonian medication 1 hour prior to the start of their appointment to ensure evaluations were completed during peak levodopa response. ${ }^{27}$ Testing was performed by a single, blinded rater, and PD motor symptoms were assessed utilizing the Movement Disorders Society Unified Parkinson's Disease Rating Scale Motor III (MDS-UPDRS). The MDS-UPDRS Motor III is the most commonly utilized rating exam to classify and assesses motor symptoms of PD with higher scores indicating greater motor symptoms. 28

Fall frequency over the past 30 days were assessed via participant recall, and individuals were prompted by study personnel asking, "How many times have you come to rest inadvertently on the ground or other lower level surface in the past 30 days?"29 Falls were also monitored during the intervention period, and participants were asked if a fall occurred at each intervention visit.

Physical activity was assessed utilizing a valid, wrist-worn activity monitor (Movband 3; DHS Group, Houston, TX) ${ }^{30}$ Participants were fitted with the activity monitor and were instructed to wear the activity monitor during all waking hours with the exception of bathing during study participation. The duration the activity monitor was worn daily was confirmed using the activity monitor's online software (Movband 3; DHS Group). Steps per day and steps per hour the device was worn (to account for varying wear times) were assessed at all 3 testing time points. In accordance with previous studies, a valid activity monitor wear day consisted of $\geq 10$ hours of wear, and valid wear weeks consisted of at least 4 valid wear days out of the 7 days of the week. ${ }^{14,31}$ Data from the activity monitors were downloaded when the participant came to the clinic for each assessment.

\section{Data Analysis}

Independent samples $t$ tests were completed to compare age, MDS-UPDRS Motor-III scores, fall frequency, disease duration, levodopa equivalent daily dose, and 2-minute walk test distance between groups (single modal and multimodal) at baseline. Tests of normality were then performed for baseline measures for all dependent variables (steps per day, steps per hour, fall frequency, and UPDRS Motor-III). UPDRS Motor-III scores (Shapiro-Wilk= $0.93, P=.20$ ) were normally distributed, but steps and falls were not (Shapiro-Wilk $\leq 0.84, P \leq .01$ ). Therefore, UPDRS Motor-III scores were analyzed using a mixed factorial, 2 groups (single modal and multimodal) by 3-time (baseline, post, and 4-wk followup) analysis of variance with repeated measures on time. Post hoc analyses on any significant effects from the analysis of variance were performed using $t$ tests. Because they were not normally distributed, nonparametric Wilcoxon signed-rank tests were utilized to compare both 30-day falls and steps (steps per day and steps per hour) across the 3 time points (baseline, post, and 4-wk post) with all participants in a single group and then again for each group (single modal and multimodal) separately. Data were analyzed using IBM SPSS statistics (version 24; IBM Corp, Armonk, NY), and significance level was set at $P \leq .05$.

\section{Results}

\section{Subject Demographics}

Two participants failed to comply with activity monitor wear time guidelines (ie, did not wear the monitor requisite days per times) and were excluded from the final analyses. Therefore, the final sample size was $\mathrm{N}=19$ (single modal $\mathrm{n}=10$, multimodal $\mathrm{n}=9$ ); those participant demographics are provided in Table 1. There were no significant differences between groups for baseline demographics related to age, disease duration, baseline MDS-UPDRS III score, levodopa equivalent daily dose, and 2-minute walk test distance.

\section{Effects of Single-Modal and Multimodal Training on Motor Scores}

Figure 2 provides MDS-UPDRS III scores for both groups across each time point. No significant main or group $\times$ time interactions were found from the mixed model $(F \leq 1.02, P>.05)$. However, there was a significant main effect of time $(F=5.68, P<.01)$ as MDS-UPDRS III scores decreased significantly from baseline (36.6 [11.2]) to posttreatment $(32.8[11.6])$ and baseline to the 4-week follow-up $(31.9[10.5])(P<.05)$, which corresponded to an $8 \%$ improvement in the single-modal group and a $15 \%$ improvement for the multimodal group. There was no evidence of

Table 1 Participant Demographics and Clinical Scores at Baseline $(\mathrm{N}=19)$

\begin{tabular}{lcc}
\hline Group & $\begin{array}{c}\text { Single modal } \\
(\mathbf{n}=\mathbf{1 0})\end{array}$ & $\begin{array}{c}\text { Multimodal } \\
(\mathbf{n}=\mathbf{9})\end{array}$ \\
\hline Gender & $9 \mathrm{M}$ & $4 \mathrm{M}$ \\
Age, y & $64.6(8.5)$ & $57.8(8.2)$ \\
Disease duration, y & $5.0(3.2)$ & $8.1(5.3)$ \\
UPDRS Motor-III score & $38.2(12.9)$ & $34.5(10.3)$ \\
Hoehn and Yahr stage & $2.3(0.5)$ & $2.2(0.4)$ \\
LEDD & $701.1(537.4)$ & $786.4(657.6)$ \\
2-Min walk test distance, $\mathrm{m}$ & $134.9(35.2)$ & $135.3(21.3)$ \\
\hline
\end{tabular}

Abbreviations: LEDD, levodopa equivalent daily dose; UPDRS, Unified Parkinson's Disease Rating Scale. Note: The values are presented as mean (SD). 


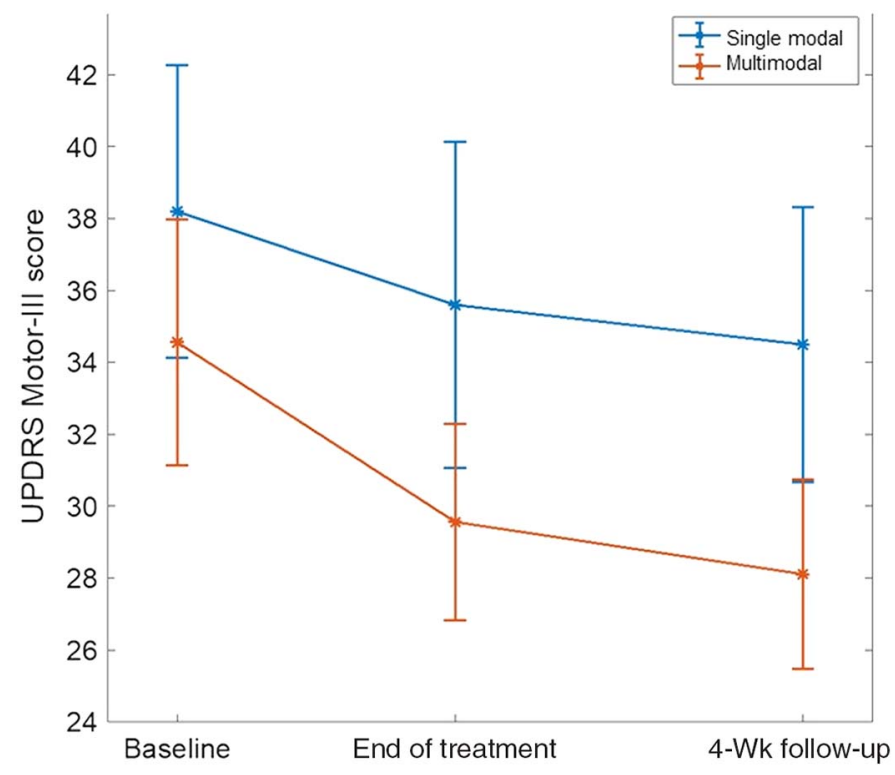

Figure 2 - Change in MDS-UPDRS Motor-III scores over time $(\mathrm{N}=19)$. UPDRS significantly decreased $(P \leq .05)$ in both the singlemodal and multimodal training groups from baseline to end of treatment, and the lower MDS-UPDRS III score was maintained through the 4-week follow-up. Data are reported as mean (SE). MDS-UPDRS indicates Movement Disorders Society Unified Parkinson's Disease Rating Scale.

difference $(P>.05)$ in MDS-UPDRS III scores from posttreatment to the 4-week follow-up.

\section{Effects of Single-Modal and Multimodal Training on Fall Frequency}

Changes in fall frequency across the 3 time points for each group separately are illustrated in Figure 3. When comparing 30-day fall

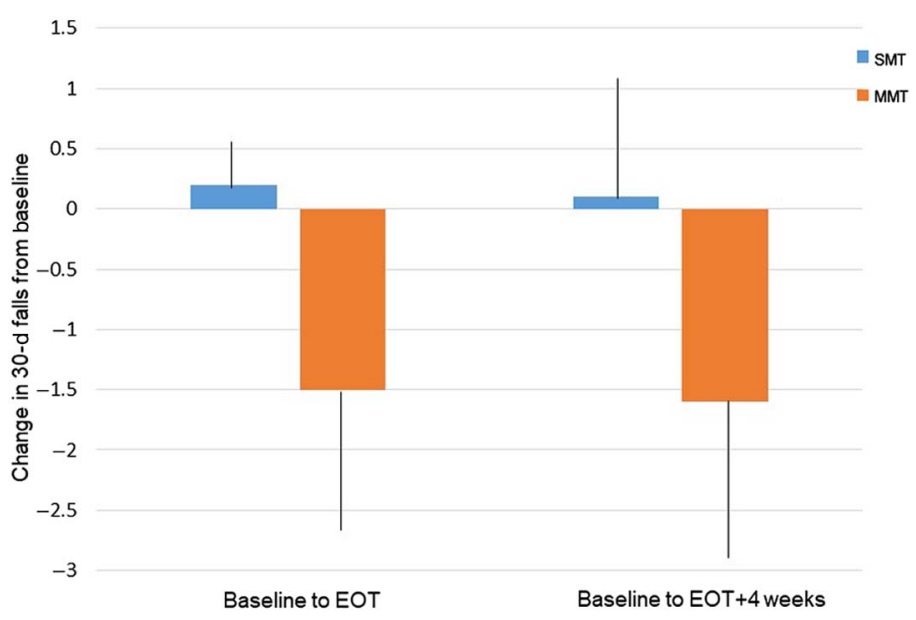

Figure 3 - Change in fall frequency over time. Only the multimodal training group significantly $(P<.02)$ decreased their number of 30-day falls from baseline to end of treatment, and the decrease in fall frequency was maintained during the 4-week follow-up. Data are reported as mean (SE). EOT indicates end of treatment; MMT, multimodal training; SMT, single-modal training. frequency across the 3 time points for all participants simultaneously, the Wilcoxon signed-rank test did not reveal any significant difference $(Z \leq 1.85, P \geq .07)$ in falls (1.5 [2.3] falls at baseline, 0.8 [1.3] falls at posttreatment, 0.7 [1.4] falls at the 4-wk follow-up). Falls also did not increase at the onset of the intervention with average 30-day falls of 1.1 (2.3) falls at intervention midpoint (4 wk after baseline). However, when analyzing the single-modal and multimodal groups separately, the multimodal group had a significant $(Z \geq 2.21, P \leq .02)$ reduction in 30-day fall frequency from baseline (2.3 [3.0] falls) to posttreatment (0.8 [1.1] falls) and from baseline to the 4-week follow-up (0.7 [1.6] falls) with no difference $(Z=0.33, P=.74)$ from posttreatment to the 4-week follow-up. There were no significant $(Z \leq 1.00, P \geq .32)$ difference in falls across the 3 time points for the single-modal group.

\section{Effects of Single-Modal and Multimodal Training on Physical Activity}

Figure 4 illustrates the steps per day increase over time for both the single-modal and multimodal training groups. When comparing steps per day across all 3 time points for all participants simultaneously, the Wilcoxon signed-rank test revealed a significant $16.5 \%$ increase $(Z=2.17, P<.05)$ in steps per day from baseline to post treatment, as mean steps per day increased from 4942 (4415) steps per day at baseline to 5914 (5425) steps per day at posttreatment. Even though, mean steps per day at the 4-week follow-up was $7.4 \%$ greater than baseline levels, no significant difference was revealed between baseline and the 4-week follow-up steps per day $(Z=1.00, P>.05)$. There was also no difference revealed in steps per day from posttreatment to the 4-week follow-up $(Z=1.19, P>.05)$. When analyzing the singlemodal and multimodal groups separately, no significant $(Z \leq .05$, $P>.05)$ differences were revealed in steps per day across the 3 time points for either the single-modal or the multimodal groups.

When comparing steps per hour across the 3 time points for all participants simultaneously, a significant $14.5 \%$ increase in steps per hour was revealed $(Z=2.21, P<.05)$ from baseline to posttreatment. Although the mean steps per hour at the 4-week

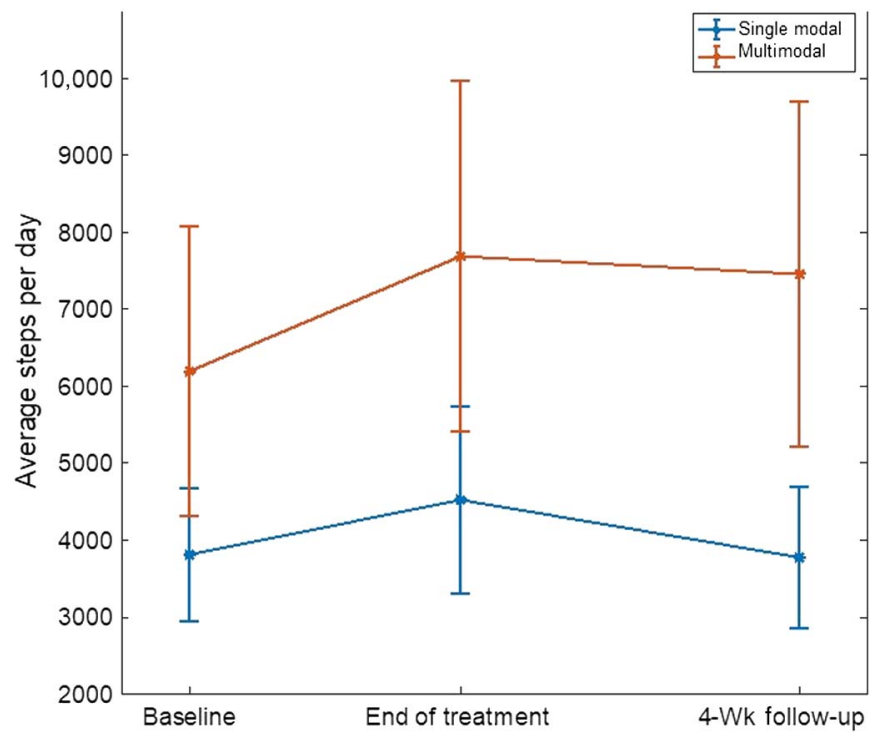

Figure 4 - Change in average steps per day over time. All individuals significantly increased their steps per day from baseline to end of treatment $(P=.03)$. Data are reported as mean $(\mathrm{SE})$. 
follow-up were $11.5 \%$ greater than baseline activity level, no significant difference was revealed $(Z=1.49, P>.05)$. Step per hour at posttreatment were not significantly different from the 4-week follow-up steps per hour $(Z=.85, P>.05)$. When analyzing the single-modal and multimodal training groups separately, no significant $(Z \leq .06, P>.05)$ differences were revealed in steps per hour across the 3 time points for neither group.

\section{Discussion}

Typically, a primary goal of exercise training in PD is to maximize motor functioning and improve quality of life. Spatial-temporal aspects of lower-extremity gait function, arm swing, and cognition as a result of single-modal and multimodal training have recently been published. ${ }^{32}$ However, another way to evaluate motor functioning, specifically mobility, is to characterize physical activity, fall frequency, and motor symptoms of PD. An important finding of this project was that although both single-modal and multimodal training increased physical activity behavior and reduced PD motor symptoms, only the multimodal group significantly decreased their fall frequency after the 8-week treatment, and the reduction in falls was maintained at the 4-week follow-up. The complex training may have facilitated or enhanced basal ganglia information processing functionality providing an environment needed for motor skill acquisition and retention, which ultimately improved automaticity even after the intervention was removed. ${ }^{23,24}$ Improvement in automaticity of a dual task, may allow for less cognitive and attention demands placed on the corticostriatal circuit, leading to more successful task completion and thus reducing fall frequency.

The reduction in fall frequency in our study differs from Strouwen et al, ${ }^{33}$ as they reported no difference between singletask and dual-task training fall frequency outcomes. The lack of difference between groups may be due to the larger sample size of the Strouwen study or their lower training frequency and duration as their participants trained twice weekly for 6 weeks compared with our greater training frequency and duration of 3 times weekly for 8 weeks.

In PD, postural instability and gait dysfunction are arguably the strongest contributor to falling. The improvement in PD motor symptoms (ie, reduced MDS-UPDRS III scores) by $8 \%$ and $15 \%$ for the single-modal and multimodal groups, respectively, may have enabled individuals to improve their gait, which may have contributed to the observed decrease in falls and increase in physical activity. ${ }^{11}$ The reduction in MDS-UPDRS III score from baseline to posttreatment across all participants exceeded the minimal clinically important difference of 3.25 on the MDSUPDRS III, and this motor symptom reduction was maintained during the 4-week follow-up period. ${ }^{34}$

For physical activity, the results of this study show a 972 step per day or nearly a $20 \%$ increase in physical activity behavior after an 8-week intervention, regardless of the mode of intervention utilized (single modal or multimodal). Current American Heart Association and American College of Sports Medicine physical activity recommendations for older adults include 30 minutes of moderate-intensity aerobic physical activity for at least 5 days per week. ${ }^{35}$ A 1000 step per day increase is equivalent to a 10 -minute bout of moderate to vigorous physical activity. ${ }^{13}$ In our study, the single-modal group increased their steps by 771 steps per day, and the multimodal group by 1495 steps per day at the end of the intervention. Baseline average steps per day in this study for the combined groups, 4942 steps per day, was similar to the 4700 PD steps per day from Benka Wallen et al. ${ }^{14}$
The present study showed nearly a 1000 steps per day increase when combining single-modal and multimodal training groups, which is larger than Conradsson et al, ${ }^{36}$ who reported an increase of 282 steps per day following a 10-week combined single-task and dual-task intervention in individuals with PD. The outcomes of the studies may differ for a number of reasons. The present intervention may have provided greater individualized attention to participants, as the intervention was administered to 1 participant at a time and only included gait training for the motor portion of the intervention. Conversely, the intervention employed by Conradsson et $\mathrm{al}^{36}$ utilized a group setting of 4 to 7 participants and included both gait and balance training. Conradsson et $\mathrm{al}^{36}$ also did not separate out single-task and dual-task trainings but rather used both training methods during the 10 -week intervention.

Although this pilot investigation was the first to report that multimodal training reduced fall frequency, it is not without limitations. The sample size was small, and although baseline fall frequency were not statistically different between the singlemodal and multimodal groups, the multimodal group did have a greater mean fall frequency at baseline, which may have allowed for a greater possibility of improvement in this outcome than the single-modal group postintervention. Also, we chose to time match the intervention groups, with both groups receiving 45-minute training sessions in lieu of dose matching interventions, which may have impacted the results. Future studies examining larger samples of participants with more similar fall histories are warranted. Finally, some individuals did take additional non-parkinsonian medications with little overlap in terms of type across groups. Non-parkinsonian medication variability and how this may impact outcomes will be taken into consideration in future trials.

\section{Conclusions}

The equivalent improvement in physical activity and PD motor symptoms in both groups suggests that single-modal and multimodal trainings may have utility in promoting physical activity and reducing PD symptoms, but only multimodal training was successful in decreasing fall frequency in individuals with PD classified as fallers. It is possible that the reduction in fall frequency in the multimodal training group was due to an improvement in basal ganglia information processing. The improvement in motor symptoms and increased physical activity behavior in both groups will likely have a positive impact upon the quality of life in individuals with PD. Therefore, although we have provided evidence of the potential utility of gait and cognitive training in individuals with $\mathrm{PD}$, additional research is warranted to determine the most efficacious mode for training.

\section{Acknowledgments}

Matthew Streicher and Sara Davidson are acknowledged for their assistance in data collection and analysis. This study was supported by a grant from the Davis Phinney Foundation. This study was registered on clinicaltrials.gov Identifier: NCT02538029. The authors have no financial disclosures to report.

\section{References}

1. Dorsey ER, Bloem BR. The Parkinson pandemic - a call to action. JAMA Neurol. 2018;75(1):9-10. PubMed ID: 29131880 doi:10.1001/ jamaneurol.2017.3299 
2. Nagano-Saito A, Martinu K, Monchi O. Function of basal ganglia in bridging cognitive and motor modules to perform an action. Front Neurosci. 2014;8:187. PubMed ID: 25071432 doi:10.3389/fnins. 2014.00187

3. Nieuwhof F, Bloem BR, Reelick MF, et al. Impaired dual tasking in Parkinson's disease is associated with reduced focusing of corticostriatal activity. Brain. 2017;140(5):1384-1398. PubMed ID: 28335024 doi:10.1093/brain/awx042

4. Bartels AL, Leenders KL. Parkinson's disease: the syndrome, the pathogenesis and pathophysiology. Cortex. 2009;45(8):915-921. PubMed ID: 19095226 doi:10.1016/j.cortex.2008.11.010

5. Wu T, Hallett M, Chan P. Motor automaticity in Parkinson's disease. Neurobiol Dis. 2015;82:226-234. PubMed ID: 26102020 doi:10. 1016/j.nbd.2015.06.014

6. Gray P, Hildebrand K. Fall risk factors in Parkinson's disease. $J$ Neurosci Nurs. 2000;32(4):222-228. PubMed ID: 10994536 doi:10. 1097/01376517-200008000-00006

7. Wielinski CL, Erickson-Davis C, Wichmann R, Walde-Douglas M, Parashos SA. Falls and injuries resulting from falls among patients with Parkinson's disease and other parkinsonian syndromes. Mov Disord. 2005;20(4):410-415. PubMed ID: 15580552 doi:10.1002/ mds. 20347

8. Bjornestad A, Tysnes OB, Larsen JP, Alves G. Loss of independence in early Parkinson disease: a 5-year population-based incident cohort study. Neurology. 2016;87(15):1599-1606. PubMed ID: 27590290 doi:10.1212/WNL.0000000000003213

9. Jasinska-Myga B, Heckman MG, Wider C, Putzke JD, Wszolek ZK, Uitti RJ. Loss of ability to work and ability to live independently in Parkinson's disease. Parkinsonism Relat Disord. 2012;18(2):130 135. PubMed ID: 21975262 doi:10.1016/j.parkreldis.2011.08.022

10. Smulders K, Dale ML, Carlson-Kuhta P, Nutt JG, Horak FB. Pharmacological treatment in Parkinson's disease: effects on gait. Parkinsonism Relat Disord. 2016;31:3-13. PubMed ID: 27461783 doi:10.1016/j.parkreldis.2016.07.006

11. Nilsson MH, Hariz GM, Iwarsson S, Hagell P. Walking ability is a major contributor to fear of falling in people with Parkinson's disease: implications for rehabilitation. Parkinsons Dis. 2012;2012:1-7. doi:10.1155/2012/713236

12. Fishman EI, Steeves JA, Zipunnikov V, et al. Association between objectively measured physical activity and mortality in NHANES. Med Sci Sports Exerc. 2016;48(7):1303-1311. PubMed ID: 26848889 doi:10.1249/MSS.0000000000000885

13. Tudor-Locke C, Craig CL, Aoyagi Y, et al. How many steps/day are enough? For older adults and special populations. Int J Behav Nutr Phys Act. 2011;8:80. PubMed ID: 21798044 doi:10.1186/1479-5868-8-80

14. Benka Wallen M, Franzen E, Nero H, Hagstromer M. Levels and patterns of physical activity and sedentary behavior in elderly people with mild to moderate Parkinson disease. Phys Ther. 2015;95(8):1135-1141. PubMed ID: 25655884 doi:10.2522/ptj. 20140374

15. Alberts JL, Linder SM, Penko AL, Lowe MJ, Phillips M. It is not about the bike, it is about the pedaling: forced exercise and Parkinson's disease. Exerc Sport Sci Rev. 2011;39(4):177-186. PubMed ID: 21799425

16. Goodwin VA, Richards SH, Taylor RS, Taylor AH, Campbell JL. The effectiveness of exercise interventions for people with Parkinson's disease: a systematic review and meta-analysis. Mov Disord. 2008;23(5):631-640. PubMed ID: 18181210 doi:10.1002/mds. 21922

17. Petzinger GM, Fisher BE, Akopian G, et al. The role of exercise in facilitating basal ganglia function in Parkinson's disease.
Neurodegener Dis Manag. 2011;1(2):157-170. PubMed ID: 23805167 doi:10.2217/nmt.11.16

18. Bloem BR, Grimbergen YA, van Dijk JG, Munneke M. The "posture second" strategy: a review of wrong priorities in Parkinson's disease. J Neurol Sci. 2006;248(1-2):196-204. doi:10.1016/j.jns. 2006.05.010

19. Rochester L, Galna B, Lord S, Burn D. The nature of dual-task interference during gait in incident Parkinson's disease. Neuroscience. 2014;265:83-94. PubMed ID: 24508154 doi:10.1016/j. neuroscience.2014.01.041

20. Yogev-Seligmann G, Rotem-Galili Y, Dickstein R, Giladi N, Hausdorff JM. Effects of explicit prioritization on dual task walking in patients with Parkinson's disease. Gait Posture. 2012;35(4):641646. PubMed ID: 22342204 doi:10.1016/j.gaitpost.2011.12.016

21. Galvan A, Devergnas A, Wichmann T. Alterations in neuronal activity in basal ganglia-thalamocortical circuits in the parkinsonian state. Front Neuroanat. 2015;9:5. PubMed ID: 25698937 doi:10. 3389/fnana.2015.00005

22. Kelly VE, Eusterbrock AJ, Shumway-Cook A. A review of dual-task walking deficits in people with Parkinson's disease: motor and cognitive contributions, mechanisms, and clinical implications. Parkinsons Dis. 2012;2012:1-15.

23. Simon DA, Bjork RA. Metacognition in motor learning. $J$ Exp Psychol Learn Mem Cogn. 2001;27(4):907-912. PubMed ID: 11486923 doi:10.1037/0278-7393.27.4.907

24. Xu T, Wang S, Lalchandani RR, Ding JB. Motor learning in animal models of Parkinson's disease: aberrant synaptic plasticity in the motor cortex. Mov Disord. 2017;32(4):487-497. PubMed ID: 28343366 doi: $10.1002 /$ mds. 26938

25. Pfeiffer E. A short portable mental status questionnaire for the assessment of organic brain deficit in elderly patients. J Am Geriatr Soc. 1975;23(10):433-441. PubMed ID: 1159263 doi:10.1111/j. 1532-5415.1975.tb00927.x

26. American College of Sports Medicine. ACSM's Guidelines for Exercise Testing and Prescription (Tenth edition). Philadelphia, PA: Wolters Kluwer; 2018.

27. Nutt JG, Woodward WR, Hammerstad JP, Carter JH, Anderson JL. The "on-off" phenomenon in Parkinson's disease. Relation to levodopa absorption and transport. N Engl J Med. 1984;310(8):483-488. PubMed ID: 6694694 doi:10.1056/NEJM198402233100802

28. Goetz CG, Tilley BC, Shaftman SR, et al. Movement disorder society-sponsored revision of the Unified Parkinson's Disease Rating Scale (MDS-UPDRS): scale presentation and clinimetric testing results. Mov Disord. 2008;23(15):2129-2170. PubMed ID: 19025984 doi: $10.1002 / \mathrm{mds} .22340$

29. Gibson MJ. The prevention of falls in later life. A report of the Kellogg International Work Group on the Prevention of Falls by the Elderly. Dan Med Bull. 1987;34(suppl 4):1-24.

30. Barkley JE, Glickman E, Fennell C, Kobak M, Williamson M, Farnell $M$. The validity of a commercially-available, low-cost, wrist-mounted accelerometer during treadmill exercise and free-living physical activity. J Sports Sci. 2019;37(7):735-740. PubMed ID: 30238836 doi:10.1080/02640414.2018.1523039

31. Trost SG, McIver KL, Pate RR. Conducting accelerometer-based activity assessments in field-based research. Med Sci Sports Exerc. 2005;37(suppl 11):S531-S543. doi:10.1249/01.mss.0000185657. 86065.98

32. Rosenfeldt AB, Penko AL, Streicher MC, Zimmerman NM, Koop MM, Alberts JL. Improvements in temporal and postural aspects of gait vary following single- and multi-modal training in individuals with Parkinson's disease [published online ahead of print May 14, 
2019]. Parkinsonism Relat Disord. doi:10.1016/j.parkreldis.2019. 05.021

33. Strouwen C, Molenaar E, Munks L, et al. Training dual tasks together or apart in Parkinson's disease: results from the DUALITY trial. Mov Disord. 2017;32(8):1201-1210. PubMed ID: 28440888 doi:10.1002/ mds. 27014

34. Horvath K, Aschermann Z, Acs P, et al. Minimal clinically important difference on the Motor Examination part of MDS-UPDRS. Parkinsonism Relat Disord. 2015;21(12):1421-1426. PubMed ID: 26578041 doi:10.1016/j.parkreldis.2015.10.006
35. Nelson ME, Rejeski WJ, Blair SN, et al. Physical activity and public health in older adults: recommendation from the American College of Sports Medicine and the American Heart Association. Med Sci Sports Exerc. 2007;39(8):1435-1445. PubMed ID: 17762378 doi:10. 1249/mss.0b013e3180616aa2

36. Conradsson D, Lofgren N, Nero H, et al. The effects of highly challenging balance training in elderly with Parkinson's disease: a randomized controlled trial. Neurorehabil Neural Repair. 2015;29(9):827-836. PubMed ID: 25608520 doi:10.1177/ 1545968314567150 